


\title{
Maschinenbautechn. Bibliothek
}

\author{
aus der Sammlung Göschen
}

Jedes Bändchen in Leinwand gebunden 90 Pfennig

Das Reohnen in der Technik und seine Hilfsmittel (Rechenschieber, Rechentafeln, Rechenmaschinen usw.) von Ingenieur Joh. Eugen Mayer. Mit 30 Abbildungen. Nr. 405.

Technisohe Tabellen und Formeln von Dr.-Ing. W. Muller, Dipl.-Ing. am Kgl. Materialprlifungsamt zu OroB-Lichterfeide. Mit 106 Figuren. Nr. 579.

Materialprufungsweson. Einfuhrung in die moderne Technik der Materialprüfung von K. Memmler, Dipl.-Ing., stăndiger Mitarbeiter am Kgl. Materialprlffungsamte $\mathrm{zu}$ GroB-Lichterfelde. I: Materialeigenschaften. - Festigkeitsversuche. - Hilfsmittel flir Festigkeitsversuche. Mit 58 Figuren. Nr. 311.

- - II : Metallprifung und Prlifung von Hilfsmaterialien des Maschinenbaues. - Baumaterialprüfung. - Papierprüfung. - Schmiermittelprüfung. - Einiges uber Metallographie. Mit 31 Figuren. Nr. 312.

Metallographle. Kurze, gemeinfaBliche Darstellung der Lehre von den Metallen und ihren Legierungen unter besonderer $\mathrm{Be}-$ rúcksichtigung der Metallmikroskopie von Prof. E. Heyn und Prof. $O$. Bauer an Kgl. Materialprufungsamt (GroB-Lichterfelde) der Kgl. Techn. Hochschule zu Berlin. I: Aligemeiner Teil. Mit 45 Abbildungen Im Text und 5 Lichtbildern auf 3 Tafeln. Nr. 432.

- II: Spezleller Teil. Mit 49 Abbildungen im Text und 37 Lichtbildern auf 19 Tafeln. Nr. 433.

Statik. I: Die Grundlehren der Statik starrer Körper von Professor W. Haubor, Diplom-Ingenieur in Stuttgart. Mit 82 Figuren. Nr. 178.

- I: Angewanate Slatik. Mit 61 Figuren. Nr. 179.

Graphlsohe Stattk mit bcoondoro Dorirksichtigung der EÍnfluBlinien von Dipl.-Ing. Otto Henkel, Oberlehrer an der Kgl. Tiefbauschule in Rendsburt. 2 Telle. Mit 207 Figuren. Nr. 603 u. 695.

FerigkeItelehre von Prof.W. Hauber, Diplom-Ingenieur in Stuttgart. Mit 56 Figuren. Nr. 288.

Anfgabensammlung zurFestigkeltslehrem.Lbeungen von R. Haren, Dipl.-Ing. in Mannheim. Mit 42 Figuren. Nr. 491.

Hydrauluk von Prof. W. Hauber, Diplom-Ingenieur in Stuttgart. Mit 44 Figuren. Nr. 397.

KInomatik von Dipl.-Ing. Hans Polster, Assistent an der Kgl.Techn. Hochschule In Dresden. Mit 76 Abbildungen. Nr. 584.

Elagtizititelehre rur Ingenieure. I; Grundlagen und Allgemelnes uber Spannungazastande, zylinder, Ebene Platten, Torsion, Gekrtimmte Trager von Prof. Dr.-Sins. Max EnBlin an der Kgl. Baugewerkschule Stuttgart und Privatdozent an der Techn. Hochschule Stuttgart. Mit $60 \mathrm{Ab}-$ blldungen. Nr. 519 . 
Geometrisohes Zelchnen von $H$. Becker, Architekt und Lehrer an der Baugewerkschule in Magdeburg, neubearbeitet von Professor J. Vonderlinn, Direktor der Kgl. Baugewerkschule in Munster. Mit 290 Figuren und 23 Tafeln im Text. Nr. 58.

Dohattenkonstruktionen von Professor J. Vonderlinn in Minster. Mit 114 Figuren. Nr. 236.

Parallelperspektive. Rechtwinklige und schiefwinklige Axonometrie von Professor J.Vonderlinn in Munster.Mit 121 Figuren. Nr.260.

Zentral - Perspektive von Architekt Hans Freyberger, neubearbeitet von Prof. J. Vonderlinn in Münster i. W. Mit 132 Fig. Nr. 57.

Darstellende Geometrie von Dr. Robert Haußner, Professor an der Universităt Jena. I. Mit 110 Figuren. Nr. 142.

- II. Mit 40 Figuren. Nr. 143.

Praktisches Maschinenzeichnen von Ing. Rich. Schiffner in Warmbrunn. I: Grundbegriffe, Einfache Maschinenteile bis zu den Kuppelungen. Mit 60 Tafeln. Nr. 589.

- II: Lager, Riem- und Seilscheiben, Zahnräder, Kolben-Pumpe, Mit 51 Tafeln. Nr. 590.

Die Masohinenelemente. KurzgefaBtes Lehrbuch mit Beispielen für das Selbststudium und den praktischen Qebrauch von Friedrich Barth, Oberingenieur in Nürnberg. Mit 86 Fig. Nr. 3.

Metallorgle von Dr. August Geitz in Kristianssand (Norwegen). I. II. Mit 21 Figuren. Nr. 313,314 .

Eisenhiftenkunde von A. KrauB, diplomierter Hutteningenieur. I: Das Roheisen. Mit 17 Figuren und 4 Tafeln. Nr. 152.

- II: Das Schmiedeisen. Mit 25 Figuren und 5 Tafeln. Nr. 153.

Lotrohrprobierkunde. Qualitative Analyse mit Hilfe des Lotrohrs von Dr.Martin Henglein in Freiberg. Mit 10 Figuren. Nr. 483.

Teohnisohe Wurmelehre (Thermodynamik) von $\mathrm{K}$. Walther und $M$. Röttinger, Diplom-lngenieuren. Mit 54 Figuren. Nr. 242.

Meohanisohe Teohnologle von Geh. Hofrat Prof. A. Lüdicke in Braunschweig. 2 Bande. Nr. $340,341$.

Die thermodynamisohen Grundlagen der Wurmekraft- und Káltemaschinen von $M$. Röttinger. DiplomIngenieur in Mannheim. Mit 73 Figuren. Nr. 2.

Dle Kalkulation $1 m$ Maschinenbau von Ingenieur $H$.Bethmann, Dozent am Technikum Altenburg. Mit 61 Abbildungen. Nr. 486.

Die Geschwindigkeitsregler der Kraftmasohinen von Dr.:9ng. H. Kröner In Friedberg. Mit 33 Figuren. Nr. 604.

Die Dampfmasohinen. Kurzgefaßtes Lehrbuch mit Beispielen flir das Selbststudium und den praktischen Gebrauch von Priedrich Barth, Oberingenieur In Nurnberg. 2 Bdchn. I: Wärmetheoretische und dampftechnische Grundlagen. Mit 64 Figuren. Nr. 8.

- II: Bau und Betrieb der Dampfmaschinen. Mit 109 Flg. Nr. 572.

Dle Damprkessel. Kurzgefaßtes Lehrbuch mit Beispielen tür das Selbststudium und den praktischen Gebrauch von Friedrich Barth, Obering. in Nüraberg I: Kesselsysteme und Feuerungen. Mit 43 Figuren. Nr. 9.

_- II : Bau und Betrieb der Dampfkessel. Mit 57 FIg. Nr.521.

Die Gaskraftmasohinen. KurzgefaBte Darstellung der wichtigsten Gasmaschinen-Bauarten von Ingenieur Alfred Kirschke. 2 Bändchen. Mit vielen Figuren. Nr. 316 u. 651 . 
Dle Dampfturbinen, ihre Wirkungsweise und Konstruktion von Ingenieur Prof. Hermann Wilda in Bremen. 3 Bandchen. Mit zahlreichen Abbildungen. Nr. 274, 715, 716.

Die Wasserturblnen von Dipl.-Ing. P. Holl in Berlin. I: Allgemeines. Die Freistrahlturbinen. Mit 113 Abbildungen. Nr. 541.

- II: Die Uberdruckturbinen. Die Wasserkraftanlagen. Mit $102 \mathrm{Ab}-$ bildungen. Nr. 542.

Die zweokmaibigste Betriebskmaft von Friedrich Barth, Oberingenieur in Nirnberg. I: Einleitung. Dampfkraftanlagen. Verschiedene Kraftmaschinen. Mit 27 Abbildungen. Nr. 224.

- II : Gas-, Wasser- und Windkraft-Anlagen. Mit 31 Abbild. Nr. 225.

- III: Elektromotoren. Betriebskostentabellen. Graphische Darstellungen. Wahl der Betriebskraft. Mit 27 Abbildungen. Nr.474.

Efeenbahnfahrzenge von $\mathrm{H}$. Hinnenthal, Kgl. Regierungsbaumeister und Oberingenieur in Hannover. I: Die Lokomotiven. Mit 89 Abbildungen im Text und 2 Tafeln. Nr. 107.

- II: Die Eisenbahnwagen und Bremsen. Mit 56 Abbildungen im Text und 3 Tafeln. Nr. 108.

Die Hebezeuge, ihre Konstruktion und Berechnung von Ingenieur Hermann Wilda, Prof. am staatl. Technikum in Bremen. Mit 399 Abbildungen. Nr. 414.

Pumpen, Druokwasser- and Druokluft-Anlagen. Ein kurzer Oberblick von Dipl.-Ing. Rudolf Vogdt, Regierungsbaumeister a. D. in Aachen. Mit 87 Abbildungen. Nr. 290.

Dielandwirtso haftliehen Masehinen von KarlWalther, Dipl.Ingenieur in Essen. 3 Bändchen. Mit vielen Abb. Nr. $407-409$.

Die Werkzeugmasohinen fur Holzbearbeitung von Ing. Prof. Herm. Wilda in Bremen. Mit 125 Abbildungen. Nr. 582.

Die Werkzeugmasohinen fir Metallbearbeltung von Ing. Prof. Hermann Wilda in Bremen. I: Die Mechanismen der Werkzeugmaschinen. Die Drehbănke. Die Frăsmaschinen. Mit 319 Abbildungen. Nr. 561.

- II: Die Bohr- und Schleifmaschinen. Die Hobel-, Shaping- und StoBmaschinen. Die Săgen und Scheren. Antrieb und Kraftbedarf. Mit 199 Abbildungen. Nr. 562.

GleBerelmaschinen von Dipl.-Ing. Emil Treiber in Heidenheim a. B. Mit 51 Figuren. Nr. 548 .

Die elektriech betriebenen Fordermasobinen von DIplom-Bergingenieur A. Balthaser. Mit vielen Fig. Nr. 678.

Die Preßluftwerkzeuge von Diplom-Ingenieur P. Iltis, Oberlehrer an der Kaiserl. Technischen Schule in Strabburg. Mit 82 Figuren. Nr. 493.

Die Baumasohinon von Ingenieur Johannes Körting in Düsseldorf. Mit 130 Abbildungen. Nr. 702 .

Tochnisches Wdrterbuoh, enthaltend die wichtigsten Ausdrücke des Maschinenbaues, Schiffbaues und der Elektrotechnik von Erich Krebs in Berlin. I: Deutsch-Englisch. Nr. 395.

- II: Englisch-Deutsch. Nr. 396.

- - III: Deutsch-Französisch. Nr. 453.

- - IV: Französisch-Deutsch. Nr. 454.

Weitere Bände erscheinen in rascher Folge. 


\section{Sammlung Göschen}

\section{Die Dampfkessel}

Kurzgefaßtes Lehrbuch

mit Beispielen für das Selbststudium

und den praktischen Gebrauch

Von

Friedrich Barth

Oberingenieur an der Baserischen Landesgewerbeanstalt in Nürnberg

\section{II \\ Bau und Betrieb der Dampfkessel}

\section{Mit 57 Figuren}

$Z$ weite, verbesserte und vermehrte Auflage

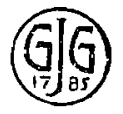

Leipzig

G. J. Göschen'sche VerlagshandIung 
Alle Rechta, instesondere das Übersetzungsrecht, ron der Verlagshandlung rorbebalten.

Druck der Spamerschen Buchdruckerei in Leipzis. 